\title{
O PAPEL DA AUTOMONITORIZAÇÃO DA GLICEMIA NOS DOENTES COM DIABETES TIPO 2 NÃO INSULINOTRATADA
}

THE ROLE OF GLUCOSE SELF-MONITORING IN NON-INSULIN-TREATED PATIENTS WITHTYPE 2 DIABETES MELITUS

Young LA, Buse JB, Weaver MA, Vu MB, Mitchell CM, Blakeney T, et al. Glucose self-monitoring in non-insulin-treated patients with type 2 diabetes in primary care settings: a randomized trial. JAMA Intern Med. 2017;177(7):920-9.

\section{Introdução}

A utilidade da automonitorização da glicemia (AMG) nos doentes com diabetes mellitus tipo 2 (DM2) não insulinotratada tem sido debatida. Diversos ensaios clínicos demonstraram benefício significativo desta medida no controlo glicémico; contudo, outros estudos não corroboraram esse efeito. Os defensores da AMG postulam que esta promove uma melhor consciencialização sobre os níveis de glicemia, levando a alterações dietéticas e no estilo de vida. Alguns ensaios verificaram que quando os doentes tinham conhecimentos adequados sobre como agir perante os diferentes resultados obtidos através da AMG, a redução da hemoglobina glicada (HbAlc) era significativamente superior comparativamente aos doentes que não dispunham dessa informação. Assim, para que a AMG seja uma ferramenta eficaz de autogestão, a díade doente-médico deveria empenhar-se ativamente em interpretar e saber como atuar sobre os valores de glicemia obtidos. Todavia, a AMG de rotina não é isenta de riscos e pode provocar consequências nefastas, nomeadamente infeção, perda de sensibilidade digital e redução da qualidade de vida $(\mathrm{QV})$.

Esta revisão tem como objetivo determinar se a AMG é benéfica nas pessoas com DM2 não insulinotratada nos cuidados de saúde primários (CSP).

\section{Métodos}

Foi realizado um ensaio clínico aleatorizado, entre janeiro de 2014 e julho de 2015, em quinze instituições de CSP localizadas na Carolina do Norte.

Os critérios de inclusão dos participantes foram: DM2 não insulinotratada, idade igual ou superior a 30 anos, HbAlc entre 6,5-9,5\% nos últimos seis meses, seguimento regular nos CSP e aceitação da inclusão em qualquer um dos grupos de estudo. Foram excluídos os doentes que pretendiam ser seguidos em consulta de endocrinologia nos doze meses seguintes, os que esta- vam medicados com insulina ou que previsivelmente iriam iniciá-la, as mulheres que planeavam engravidar nos doze meses após o início do estudo e os doentes que tencionavam mudar de local de residência.

Os participantes foram aleatorizados e distribuídos por três grupos, com três abordagens diferentes: $1^{\circ}$ grupo, sem AMG; $2^{\circ}$ grupo, AMG uma vez por dia; $3^{\circ}$ grupo, AMG uma vez por dia com receção de mensagens automatizadas e personalizadas através de um glucómetro Telcare $\odot$, destinadas a educar e motivar os doentes a controlar os valores de glicemia.

O objetivo primário era avaliar os níveis de HbAlc e de qualidade de vida $(\mathrm{QV})$ (vertente física e mental, medidas através da aplicação do questionário SF-36) no início do estudo e após 52 semanas. A frequência e gravidade de hipoglicemias, a recorrência ao serviço de urgência ou internamentos relacionados com a diabetes foram objetivos secundários do estudo.

\section{Resultados}

Foram aleatorizados 450 doentes com DM2 não insulinotratada pelos três grupos, dos quais $92,9 \%$ $(n=418)$ completou o estudo. A idade média dos participantes foi de 61 anos, sendo o tempo médio de diagnóstico da diabetes de oito anos. Não se verificou diferença entre os três grupos quanto à preferência dos doentes relativamente à utilização de AMG (globalmente $22 \%$ preferia não realizá-la, enquanto $40 \%$ gostaria de a efetuar). Relativamente aos antidiabéticos orais, a maioria (80\%) dos participantes estava medicado com metformina, sendo as sulfonilureias o segundo grupo farmacológico mais utilizado (35\%).

Após um ano de seguimento não foram observadas diferenças significativas nos níveis de HbAlc entre os participantes do grupo sem AMG e os restantes $(P=0,74)$. Também não houve diferença estatisticamente significativa na $\mathrm{QV}$, quer a nível físico $(P=0,48)$ quer a nível mental $(P=0,90)$. Relativamente aos prin- 
cipais eventos adversos, nomeadamente a frequência e gravidade de hipoglicemia, à utilização dos cuidados de saúde ou à iniciação da insulina, não se verificaram diferenças notáveis entre os três grupos.

Foram realizadas análises de subgrupo para as seguintes variáveis: realização de AMG prévia ao estudo, duração da DM2, valor de HbAlc no início do estudo, utilização de fármacos secretagogos de insulina, comorbilidades, idade, raça e escolaridade. Não se verificou diferença significativa no controlo glicémico ajustado para as diferentes variáveis. Quanto à vertente física da QV observou-se que os indivíduos de raça afro-americana alocados ao $3^{\circ}$ grupo (AMG uma vez por dia, com receção de mensagens através do glucómetro Telcare() reportaram menor QV relativamente ao grupo sem AMG $(P=0,02)$. Não se registaram outras diferenças significativas no que concerne à $\mathrm{QV}$.

Quanto à adesão dos participantes ao grupo alocado apurou-se que, no grupo sem AMG, 23,7\% ( $n=36)$ dos doentes reportaram que realizaram diversas AMG por mês, $1 \%(n=2)$ realizou uma AMG por mês e 8,5\% $(n=13)$ referiu efetuar AMG esporádica (menos do que uma vez por mês) durante o período em estudo.

\section{Conclusão}

Não foram observadas diferenças estatisticamente significativas nos níveis de HbAlc ou QV entre os doentes com DM2 não insulinotratada que realizaram AMG comparativamente com os que não a efetuaram.

A adição de mensagens automatizadas não proporcionou nenhuma vantagem no controlo glicémico após um ano de seguimento. Todavia, os autores reforçam que o viés de participação pode estar na base da ausência de benefício das mensagens automatizadas, uma vez que os participantes poderão ser indivíduos com maior preocupação com o controlo da sua doença do que a população geral.

Assim, os resultados deste estudo sugerem que a AMF não deve ser aconselhada por rotina em doentes com DM2 não insulinotratada.

\section{Comentário}

A importância da AMG nos doentes com diabetes mellitus tipo 1 (DM1) e nos doentes com DM2 medicados com insulina é indiscutível, uma vez que para além de reduzir o risco de hipoglicemias graves diminui também a incidência de complicações micro e macrovasculares. ${ }^{1-2} \mathrm{~A}$ informação sobre os níveis plasmáticos de glicose permite o ajuste da dose de insulina, contribuindo para um controlo glicémico mais eficaz. ${ }^{3}$

Quanto aos doentes com DM2 não insulinotratada, o papel da AMG não é consensual. Enquanto uns estudos advogam a sua importância, tendo em conta a redução verificada nos níveis de $\operatorname{HbAlc~}(-0,2$ a $-0,3 \%),{ }^{4-5}$ outros defendem que esta diminuição é modesta e não é clinicamente relevante. ${ }^{6}$ Contudo, diversos estudos recentes não encontraram melhoria do controlo glicémico ou satisfação dos doentes nem redução das complicações da doença..$^{7-8}$

No início do ensaio clínico em análise, todos os participantes receberam folhetos informativos sobre os valores alvo de glicemia e os sintomas sugestivos de hipo e hiperglicemia. Após a aleatorização, todos os doentes tiveram uma consulta médica onde lhes foram dadas indicações clínicas acerca da DM2 e de como gerir a doença. Contudo, depois de um ano de seguimento, a AMG não surtiu efeito benéfico nos níveis de HbA1c ou na QV dos doentes com DM2 não insulinotratada. Apesar de algumas revisões prévias reportarem que a utilização de meios tecnológicos poderia aumentar a adesão dos doentes à terapêutica e, consequentemente, ao controlo da doença, este estudo concluiu que a adição de mensagens personalizadas, com vista a motivar e orientar os doentes, não beneficiou o controlo glicémico.

Relativamente às limitações deste ensaio clínico salienta-se o facto de o processo de aleatorização ser aberto, pois os participantes e investigadores tiveram conhecimento da intervenção aplicada. Além disso, na prática clínica os doentes com diabetes têm uma vigilância mais apertada, com uma periodicidade de consultas ajustada ao grau de controlo me- 
tabólico. Esta maior proximidade médico-doente, assim como a interpretação conjunta dos valores glicémicos obtidos através da AMG, poderia resultar em ajustes terapêuticos mais precoces e, consequentemente, num melhor controlo glicémico. Dado que neste estudo não houve visitas de seguimento durante o período de um ano, este pode constituir um viés importante. Destaca-se ainda o viés de participação, uma vez que os doentes que aceitaram participar no estudo poderão ser diferentes dos que não aceitaram (nomeadamente ter maior capacidade de autogestão da doença e maior motivação para atingir o controlo glicémico) e o facto de nem todos os participantes terem aderido completamente ao grupo ao qual foram atribuídos por aleatorização.

Tendo em conta que os ensaios clínicos realizados até à data apresentam diferentes metodologias e limitações inerentes, a relevância da AMG nos doentes com DM2 não insulinotratada continua por esclarecer e o significado clínico da ligeira redução da HbAlc, reportada por alguns estudos, permanece incerto. Consequentemente, a posição assumida pelas diversas associações internacionais, nomeadamente a American Diabetes Association e a International Diabetes Federation, tem variado ao longo dos últimos anos.

De acordo com a evidência disponível, a AMG não deve ser recomendada por rotina neste subgrupo de doentes, uma vez que não parece melhorar os resultados em saúde desta população, não sendo, portanto, custo-efetiva. Todavia, esta medida pode ser benéfica aquando do início ou titulação da dose de um antidiabético oral com potencial risco de hipoglicemias, pelo que deverá ser ponderada individualmente, baseando-se num processo de decisão partilhada entre o doente com diabetes e o seu médico assistente.
Pedro Namora

Médico Interno de Medicina Geral e Familiar. USF Famalicão I - ACeS Ave-Famalicão Salete Gomez

Médica Interna de Medicina Geral e Familiar. USF Fafe Sentinela - ACeS do Alto Ave

\section{REFERÊNCIAS BIBLIOGRÁFICAS}

1. Nathan DM, Cleary PA, Backlund JY, Genuth SM, Lachin JM, Orchard $\mathrm{T}$, et al. Intensive diabetes treatment and cardiovascular disease in patients with type 1 diabetes. N Engl J Med. 2005;353(25):264353.

2. American Diabetes Association. Standards of medical care in diabetes - 2016. Diabetes Care. 2016;39 Suppl 1.

3. Welschen LM, Bloemendal E, Nijpels G, Dekker JM, Heine RJ, Stalman WA, et al. Self-monitoring of blood glucose in patients with type 2 diabetes who are not using insulin. Cochrane Database Syst Rev. 2005;(2):CD005060.

4. Poolsup N, Suksomboon N, Rattanasookchit S. Meta-analysis of the benefits of self-monitoring of blood glucose on glycemic control in type 2 diabetes patients: an update. Diabetes Technol Ther. 2009;11(12):775-84.

5. Barnett AH, Krentz AJ, Strojek K, Sieradzki J, Azizi F, Embong M, et al. The efficacy of self-monitoring of blood glucose in the management of patients with type 2 diabetes treated with a gliclazide modified release-based regimen: a multicentre, randomized, parallel-group, 6-month evaluation (DINAMIC 1 study). Diabetes Obes Metab. 2008;10(12):1239-47.

6. Farmer AJ, Perera R, Ward A, Heneghan C, Oke J, Barnett AH, et al. Meta-analysis of individual patient data in randomised trials of self-monitoring of blood glucose in people with non-insulin treated type 2 diabetes. BMJ. 2012;344:e486.

7. Malanda UL, Welschen LM, Riphagen II, Dekker JM, Nijpels G, Bot SD. Self-monitoring of blood glucose in patients with type 2 diabetes mellitus who are not using insulin. Cochrane Database Syst Rev. 2012;1:CD005060.

8. Kleefstra N, Hortensius J, Logtenberg SJ, Slingerland RJ, Groenier KH, Houweling ST, et al. Self-monitoring of blood glucose in tablettreated type 2 diabetic patients (ZODIAC). Neth J Med. 2010;68(1): 311-6.

\section{CONFLITOS DE INTERESSE}

Os autores declaram não ter quaisquer conflitos de interesse. 